\title{
VASCULAR FLORA OF THE NATURA 2000 AREA “ŁĄKI NAD WOJKÓWKĄ" PLH 180051 (DYNOWSKIE FOOTHILLS)
}

\author{
MARia Ziaja, TOMASZ WÓJCIK
}

M. Ziaja, T. Wójcik, Department of Ecology and Nature Protection, University of Rzeszów, Cicha 2 A, 35-326 Rzeszów, Poland, e-mail: mziaja@ur.edu.pl, antomi7@wp.pl

(Received: March 27, 2015. Accepted: May 8, 2015)

\begin{abstract}
AвSTRAct. The article presents the results of floristic investigations conducted in 2012-2014 in the Natura 2000 area "Łąki nad Wojkówką" (PLH 180051) situated in the Dynowskie Foothills. The area was established with the aim of conservation of thermophilic meadow complexes with fragments of xerothermic grasslands, which are extremely rare in the Carpathians. The vascular flora comprises 252 species from 49 families and 166 genera. Native spontaneophytes $(45.7 \%)$ and apophytes $(44.8 \%)$ dominated over anthropophytes $(9.5 \%)$, and perennial species $(85.3 \%)$ dominated over short-lived $(14.7 \%)$ species. Thermophilic species of dry grasslands and scrubs were represented by the classes Festuco-Brometea, Trifolio-Geranietea, and Rhamno-Prunetea. Fresh meadow plants from the class Molinio-Arrhenatheretea were most frequently noted in the flora composition, while ruderal species from the class Artemisietea vulgaris had a substantial share. The occurrence of protected species e.g. Centaurium erythraea, Dianthus armeria, and Gentiana cruciata should also be noted.
\end{abstract}

KEY WORDs: vascular flora, “Łąki nad Wojkówką” Natura 2000 PLH 180051, xerothermic grassland, Western Carpathians, Podkarpackie Province

\section{INTRODUCTION}

The Natura 2000 area "Łąki nad Wojkówką" (PLH 180051) protects fragments of the thermophilic xerothermic plant flora, which is rare in the Carpathian Foothills.

In the Carpathians, xerothermic grasslands represent extremely rare communities distributed as insular localities primarily in the Pieniny Mts. (KaźmierczaKowa 2004) and in the Przemyskie Foothills (KuCHARZYK 2010, Trąi et al. 2012). Fresh thermophilic meadows, which have been described in the Strzyżowskie Foothills (Towpasz 1990, WójciK \& PiĄTEK 2015), Przemyskie Foothills (BARABASZ-KRASNY 2011), and Dynowskie Foothills (ZiAja \& WóJCiK 2014), occur more frequently.

Xerothermic grasslands are one of the most valuable but simultaneously most vulnerable elements of the natural habitats in Poland and Europe; hence, they have been listed in Annex I of the European Union Habitats Directive (Council Directive... 1992) as a special priority habitat. This was prompted by the limited range of their occurrence, considerable frag- mentation, and abandonment of agricultural management thereof, which is an important factor in the development of these habitats (PerzanowsKa \& KuJAWA-PAWLACZYK 2004).

The aim of the study was to make an inventory and analyse the vascular flora of the Natura 2000 area "Łąki nad Wojkówką" established to conserve the xerothermic flora.

\section{STUDY AREA}

The Natura 2000 area "Łąki nad Wojkówką" (PLH 180051) comprises three grassland-meadow complexes (Fig. 1) with a total area of 9.6 ha situated on the hills of the Wisłok River valley (Mróz \& Rogata 2011). In the administrative division, the area is located in the Podkarpackie Province, Krosno Poviat, and Wojaszówka Commune. The entire area is part of the Czarnorzecko-Strzyżowski Landscape Park. According to the physical-geographical division proposed by KONDRACKI (2011), the analysed area is situated in two mezoregions: Jasielsko-Krośnieńska Basin and Dynowskie Foothills, which are part of 


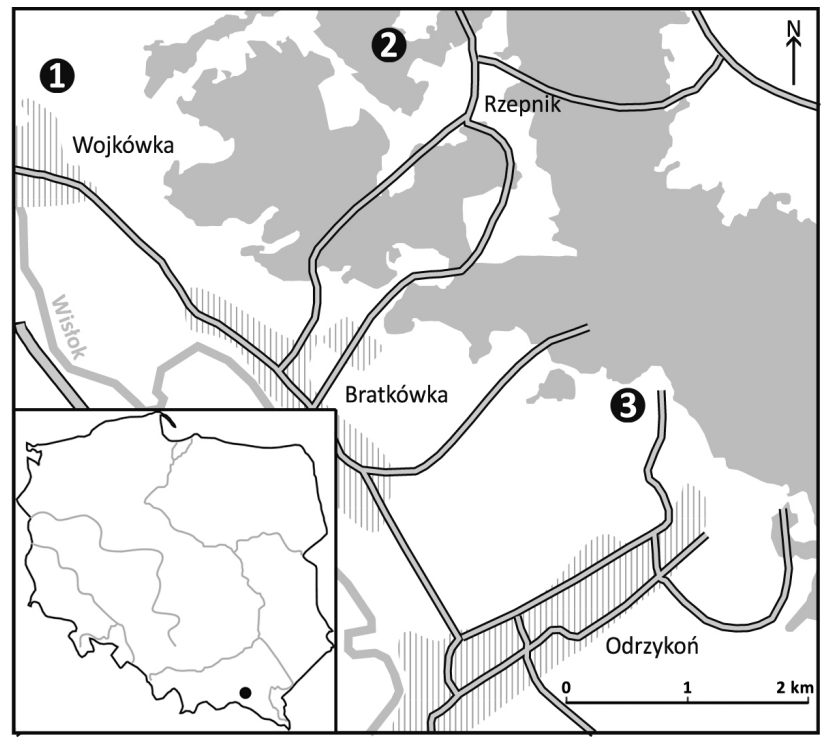

Fig. 1. Location of study area: 1 - Wojkówka, 2 - Rzepnik, 3 - Odrzykoń

the Środkowobeskidzkie Foothills macroregion, Outer Western Carpathians subprovince, and Western Carpathians Province with the Subcarpathia. In accordance with the geobotanical division of Poland (Pawıowski 1977), the area is located in the Fliszowe Foothills subregion, Western Carpathians subdivision, Carpathian division, Alpine subprovince.

The locality in Wojkówka is situated on the S-SW slopes of Ptasznik Hill (369 m a.s.l.); it covers a steep scarp over a road and the top part of a quarry. The thermophilic grasslands here are adjacent to arable land. The locality is only partially grazed, whereas the scarp over the road and the top part of the quarry are largely overgrown by trees and shrubs. In Odrzykon, the vegetation forms a mosaic of grasslands and thermophilic scrubs growing on the S slope of Piekło Hill (386 $\mathrm{m}$ a.s.l.). The nearby abandoned quarry is also a part of the area. Currently, a vast part of the area is overgrown by scrubs from the class Rhamno-Prunetea. Meadows and grasslands occupy only small enclaves mainly located at the scrub edges and within the quarry. The third area situated in Rzepnik comprises an isolated clearing surrounded by forests on the $S$ slope of Kiczary Hill (438 m a.s.l.). For several years, the area has not been managed, which has resulted in unfavourable changes in the plant communities. Phytocoenoses from the class Festuco-Brometea, occupying approx. $20 \%$ of the total area, are located in the central part of the analysed site on a fragment of a slope with the highest inclination of ca. $45^{\circ}$. The other part is covered by meadows from the class Molinio-Arrhenatheretea, which have undergone transformation as well (Mróz \& RogałA 2011, ZiAjA \& WóJCIK 2014). A greater part of the grassland area has been dominated by high perennials, e.g. Solidago gigantea, Calamagrostis epigejos in insolated and dry areas and by Mentha longifolia, Urtica dioica, Juncus inflexus in wetter fragments as well as ruderal species, e.g. Bromus inermis and Cirsium arvense. In general, stands occupied by one or several of the aforementioned species are predominant, but there are no stands typical for fresh meadows and xerothermic grasslands.

\section{MATERIALS AND METHODS}

The field investigations were carried out during the vegetation season in 2012-2014 over the entire Natura 2000 area "Łąki nad Wojkówką", and more specifically in three localities: Wojkówka, Odrzykoń, and Rzepnik. The nomenclature for the plant species followed that proposed by MireK et al. (2002) and the family names were used after RUtKowsкi (2004). In the analysis of the flora based on the categories of life forms described by Raunkiaer (ZARZYCKI et al. 2002), affiliation to geographical-historical groups was determined and native species were divided into non-synanthropic spontaneophytes and apophytes (synanthropic spontaneophytes) (KoRnAś 1968, ZAJĄC 1979, ZAJĄC \& ZająC 1992, 2011, TOKARSKA-GuZIK 2005). Additionally, phytosociological affiliation (Matuszkiewicz 2005) was provided and the status of the protected plants was presented based on the Regulation (RoZPORZĄDZENIE... 2014).

\section{RESULTS}

In total, 252 species of vascular plants from 166 genera and 49 families were noted in the analysed area (Table 1). Families represented by the most numerous species included Asteraceae (29 species - 11.5\%), Poaceae (28 species - 11.1\%), Fabaceae (24 species $-9.5 \%)$, Rosaceae (20 species $-8 \%$ ), Lamiaceae (17 species $-6.7 \%$ ), and Apiaceae (11 species - 4.4\%). Altogether there were 129 species, which accounted for $51.2 \%$ of the flora. Other 13 families were represented by one species and nine families by two species.

The flora of the "Łąki nad Wojkówką" area comprised $63.5 \%$ of hemicryptophytes (160 species) and a substantial share of therophytes $14.7 \%$ (37 species). The proportion of representatives of other life forms was clearly lower, i.e. $8.7 \%$ of geophytes (22 species), $5.6 \%$ of megaphanerophytes (14 species), $5.1 \%$ of nanophanerophytes (13 species), and $2.4 \%$ of chamephytes (6 species) (Table 2 ).

As many as $27(10.7 \%)$ representatives of dendroflora were noted in the analysed area, which is caused by progressive succession and the close neighbourhood of forests. These species usually occurred singly or formed small clusters. Quercus robur, Populus tremula, Fraxinus excelsior, Acer campestre, Prunus spinosa, Crataegus monogyna, Rosa canina, and Ligustrum vulgare were the most abundant species.

A vast majority of the analysed flora were native species (228 species $-90.5 \%$ ), including 148 apo- 
Table 1. List of vascular plant species found in the Natura 2000 "Łąki nad Wojkówką"

\begin{tabular}{|c|c|c|c|c|c|c|c|c|}
\hline \multirow{2}{*}{ No. } & \multirow{2}{*}{ Name of species } & \multirow{2}{*}{ Family } & \multicolumn{3}{|c|}{ Locality } & \multirow{2}{*}{ LF } & \multirow{2}{*}{ GHg } & \multirow{2}{*}{ PSg } \\
\hline & & & W & $\mathrm{O}$ & $\mathrm{R}$ & & & \\
\hline 1 & Abies alba Mill. & Pinaceae & & & + & M & $\mathrm{Sp}$ & Vac-Pice \\
\hline 2 & Acer campestre $\mathrm{L}$. & Aceraceae & + & + & + & M & $\mathrm{Sp}$ & Que-Fag \\
\hline 3 & Achillea millefolium L. s. str. & Asteraceae & + & + & + & $\mathrm{H}$ & Ap & Mol-Arr \\
\hline 4 & Aegopodium podagraria $\mathrm{L}$. & Apiaceae & + & + & + & G & Ap & Que-Fag \\
\hline 5 & Agrimonia eupatoria L. & Rosaceae & + & + & + & $\mathrm{H}$ & Ap & Trif-Ger \\
\hline 6 & Agrostis capillaris L. & Poaceae & & + & + & $\mathrm{H}$ & Ap & Nard-Cal \\
\hline 7 & Ajuga genevensis L. & Lamiaceae & + & & & $\mathrm{H}$ & $\mathrm{Sp}$ & Fest-Brom \\
\hline 8 & Ajuga reptans $\mathrm{L}$. & Lamiaceae & & & + & $\mathrm{H}$ & $\mathrm{Sp}$ & - \\
\hline 9 & Alchemilla monticola Opiz & Rosaceae & + & + & & $\mathrm{H}$ & $\mathrm{Sp}$ & Mol-Arr \\
\hline 10 & Allium oleraceum $\mathrm{L}$. & Alliaceae & + & + & + & G & $\mathrm{Sp}$ & Fest-Brom \\
\hline 11 & Allium vineale $\mathrm{L}$. & Alliaceae & + & + & + & G & Ap & - \\
\hline 12 & Alnus glutinosa (L.) Gaertn. & Betulaceae & & & + & M & Ap & Que-Fag \\
\hline 13 & Alnus incana (L.) Moench & Betulaceae & & & + & M & Ap & Que-Fag \\
\hline 14 & Anagallis arvensis $\mathrm{L}$. & Primulaceae & + & & & $\mathrm{T}$ & Arch & Stel med \\
\hline 15 & Angelica sylvestris $\mathrm{L}$. & Apiaceae & + & + & + & $\mathrm{H}$ & Ap & Mol-Arr \\
\hline 16 & Anthoxanthum odoratum L. & Poaceae & + & + & + & $\mathrm{H}$ & Ap & - \\
\hline 17 & Anthriscus sylvestris (L.) Hoffm. & Apiaceae & + & & & $\mathrm{H}$ & Ap & Artemi \\
\hline 18 & Arabis glabra (L.) Bernh. & Brassicaceae & + & + & & $\mathrm{H}$ & $\mathrm{Sp}$ & - \\
\hline 19 & Arabis hirsuta (L.) Scop. & Brassicaceae & & + & & $\mathrm{H}$ & Sp & Fest-Brom \\
\hline 20 & Arenaria serpyllifolia $\mathrm{L}$. & Caryophyllaceae & + & & & $\mathrm{T}$ & Ap & - \\
\hline 21 & $\begin{array}{l}\text { Arrhenatherum elatius (L.) Beauv. ex J. Presl } \\
\text { et C. Presl }\end{array}$ & Poaceae & + & + & + & $\mathrm{H}$ & Ap & Mol-Arr \\
\hline 22 & Artemisia vulgaris L. & Asteraceae & + & + & + & $\mathrm{H}$ & Ap & Artemi \\
\hline 23 & Asarum europaeum L. & Aristolochiaceae & + & + & + & $\mathrm{H}$ & $\mathrm{Sp}$ & Que-Fag \\
\hline 24 & Astragalus glycyphyllos L. & Fabaceae & & + & & $\mathrm{H}$ & Ap & Trif-Ger \\
\hline 25 & Ballota nigra $\mathrm{L}$. & Lamiaceae & + & & & $\mathrm{H}$ & Arch & Artemi \\
\hline 26 & Barbarea vulgaris R. Br. & Brassicaceae & + & & & $\mathrm{H}$ & Ap & - \\
\hline 27 & Betonica officinalis L. & Brassicaceae & & + & + & $\mathrm{H}$ & $\mathrm{Sp}$ & Mol-Arr \\
\hline 28 & Betula pendula Roth & Betulaceae & + & + & + & M & Ap & Rham-Pru \\
\hline 29 & Bidens frondosa $\mathrm{L}$. & Asteraceae & & & + & $\mathrm{T}$ & $\mathrm{Kn}$ & Bident \\
\hline 30 & Briza media L. & Poaceae & + & + & + & $\mathrm{H}$ & $\mathrm{Sp}$ & - \\
\hline 31 & Bromus inermis Leyss. & Poaceae & + & & + & $\mathrm{H}$ & Ap & Fest-Brom \\
\hline 32 & Calamagrostis epigejos (L.) Roth & Poaceae & + & + & + & G & Ap & Epilob \\
\hline 33 & Campanula patula $\mathrm{L}$. & Campanulaceae & & & + & $\mathrm{H}$ & Ap & Mol-Arr \\
\hline 34 & Campanula rapunculoides $\mathrm{L}$. & Campanulaceae & & + & + & $\mathrm{H}$ & Ap & Trif-Ger \\
\hline 35 & Campanula trachelium L. & Campanulaceae & + & + & & $\mathrm{H}$ & $\mathrm{Sp}$ & Que-Fag \\
\hline 36 & Carduus acenthoides L. & Asteraceae & + & + & + & $\mathrm{H}$ & Arch & Artemi \\
\hline 37 & Carex caryophyllea Latourr. & Cyperaceae & + & + & & $\mathrm{G}, \mathrm{H}$ & Sp & Fest-Brom \\
\hline 38 & Carex hirta L. & Cyperaceae & + & + & + & $\mathrm{G}$ & Ap & Mol-Arr \\
\hline 39 & Carex pairae F.W. Schultz & Cyperaceae & & + & & $\mathrm{H}$ & Ap & - \\
\hline 40 & Carex pilulifera $\mathrm{L}$. & Cyperaceae & + & & & $\mathrm{H}$ & $\mathrm{Sp}$ & Nard-Cal \\
\hline 41 & Carex spicata Huds. & Cyperaceae & + & + & & $\mathrm{H}$ & Ap & - \\
\hline 42 & Carex sylvatica Huds. & Cyperaceae & & & + & $\mathrm{H}$ & $\mathrm{Sp}$ & Que-Fag \\
\hline 43 & Carex tomentosa $\mathrm{L}$. & Cyperaceae & & & + & $\mathrm{G}, \mathrm{H}$ & $\mathrm{Sp}$ & Mol-Arr \\
\hline 44 & Carlina vulgaris $\mathrm{L}$. & Asteraceae & + & + & + & $\mathrm{H}$ & Ap & Fest-Brom \\
\hline 45 & Centaurea jacea L. & Asteraceae & + & + & + & $\mathrm{H}$ & Ap & Mol-Arr \\
\hline 46 & Centaurea scabiosa L. & Asteraceae & + & + & + & $\mathrm{H}$ & $\mathrm{Sp}$ & Fest-Brom \\
\hline 47 & Centaurium erythraea Rafn [P] & Gentianaceae & + & & + & $\mathrm{T}$ & $\mathrm{Sp}$ & Epilob \\
\hline 48 & Cerasus avium (L.) Moench & Rosaceae & + & + & + & M & Ap & Que-Fag \\
\hline 49 & Cerinthe minor $\mathrm{L}$. & Boraginaceae & + & & & $\mathrm{H}$ & Ap & - \\
\hline 50 & Chaerophyllum aromaticum $\mathrm{L}$. & Apiaceae & + & + & + & $\mathrm{H}$ & Ap & Artemi \\
\hline 51 & Chaerophyllum bulbosum $\mathrm{L}$. & Apiaceae & + & + & & $\mathrm{T}, \mathrm{G}$ & Ap & Artemi \\
\hline 52 & Chamaenerion angustifolium (L.) Scop. & Onagraceae & + & & & $\mathrm{H}$ & Ap & Epilob \\
\hline 53 & Cichorium intybus L. & Asteraceae & + & + & + & $\mathrm{H}$ & Arch & Artemi \\
\hline 54 & Circaea lutetiana L. & Onagraceae & & & + & G & Sp & Que-Fag \\
\hline
\end{tabular}




\begin{tabular}{|c|c|c|c|c|c|c|c|c|}
\hline \multirow{2}{*}{ No. } & \multirow{2}{*}{ Name of species } & \multirow{2}{*}{ Family } & \multicolumn{3}{|c|}{ Locality } & \multirow{2}{*}{ LF } & \multirow{2}{*}{ GHg } & \multirow{2}{*}{ PSg } \\
\hline & & & $\mathrm{W}$ & $\mathrm{O}$ & $\mathrm{R}$ & & & \\
\hline 55 & Cirsium arvense (L.) Scop. & Asteraceae & + & + & + & G & Ap & Artemi \\
\hline 56 & Cirsium oleraceum (L.) Scop. & Asteraceae & & & + & $\mathrm{H}$ & Ap & Mol-Arr \\
\hline 57 & Cirsium rivulare (JACQ.) All. & Asteraceae & & & + & $\mathrm{H}$ & $\mathrm{Sp}$ & Mol-Arr \\
\hline 58 & Clinopodium vulgare $\mathrm{L}$. & Lamiaceae & + & + & + & $\mathrm{H}$ & $\mathrm{Sp}$ & Trif-Ger \\
\hline 59 & Consolida regalis Gray & Ranunculaceae & + & + & & $\mathrm{T}$ & Arch & Stel med \\
\hline 60 & Convolvulus arvensis $\mathrm{L}$. & Convolvulaceae & + & + & + & $\mathrm{H}$ & Ap & Agr-rep \\
\hline 61 & Cornus sanguinea $\mathrm{L}$. & Cornaceae & + & + & + & $\mathrm{N}$ & Ap & Rham-Pru \\
\hline 62 & Coronilla varia $\mathrm{L}$. & Fabaceae & + & + & + & $\mathrm{H}$ & Ap & Trif-Ger \\
\hline 63 & Crataegus monogyna Jacq. & Rosaceae & + & + & + & $\mathrm{N}$ & Ap & Rham-Pru \\
\hline 64 & Crepis biennis L. & Asteraceae & + & + & + & $\mathrm{H}$ & Ap & Mol-Arr \\
\hline 65 & Cruciata glabra (L.) Ehrend. & Rubiaceae & + & + & + & $\mathrm{H}$ & $\mathrm{Sp}$ & Que-Fag \\
\hline 66 & Cuscuta epithymum (L.) L. s. str. & Cuscutaceae & + & + & + & $\mathrm{T}$ & $\mathrm{Sp}$ & Nard-Cal \\
\hline 67 & Cuscuta europaea L. & Cuscutaceae & + & & & $\mathrm{T}$ & $\mathrm{Sp}$ & Artemi \\
\hline 68 & Cynosurus cristatus L. & Poaceae & + & & & $\mathrm{H}$ & $\mathrm{Sp}$ & Mol-Arr \\
\hline 69 & Dactylis glomerata L. & Poaceae & + & + & + & $\mathrm{H}$ & Ap & Mol-Arr \\
\hline 70 & Danthonia decumbens DC. & Poaceae & & + & & $\mathrm{H}$ & Sp & Nard-Cal \\
\hline 71 & Daucus carota L. & Apiaceae & + & + & + & $\mathrm{H}$ & Ap & Mol-Arr \\
\hline 72 & Deschampsia caespitosa (L.) P. Beauv. & Poaceae & & + & + & $\mathrm{H}$ & Sp & Mol-Arr \\
\hline 73 & Dianthus armeria $\mathrm{L} .[\mathrm{S}]$ & Caryophyllaceae & & & + & $\mathrm{H}$ & $\mathrm{Sp}$ & - \\
\hline 74 & Dianthus deltoides L. & Caryophyllaceae & & + & & $\mathrm{H}$ & Sp & Koel-Cory \\
\hline 75 & Dipsacus sylvestris Huds. & Dipsacaceae & + & & & $\mathrm{H}$ & Ap & Artemi \\
\hline 76 & Dryopteris carthusiana (Vill.) H. P. Fuchs & Dryopteridaceae & + & & + & $\mathrm{H}$ & $\mathrm{Sp}$ & - \\
\hline 77 & Dryopteris filix-mas (L.) Schott & Dryopteridaceae & & & + & $\mathrm{H}$ & $\mathrm{Sp}$ & Que-Fag \\
\hline 78 & Echium vulgare $\mathrm{L}$. & Boraginaceae & + & + & & $\mathrm{H}$ & Ap & Artemi \\
\hline 79 & Elymus repens (L.) Gould & Poaceae & + & + & + & G & Ap & Agr-rep \\
\hline 80 & Equisetum arvense $\mathrm{L}$. & Equisetaceae & + & + & + & G & Ap & Agr-rep \\
\hline 81 & Equisetum sylvaticum L. & Equisetaceae & & & + & G & $\mathrm{Sp}$ & - \\
\hline 82 & Equisetum telmateia Ehrh. & Equisetaceae & & + & + & G & $\mathrm{Sp}$ & Que-Fag \\
\hline 83 & Erigeron acer $\mathrm{L}$. & Asteraceae & + & & + & $\mathrm{H}, \mathrm{T}$ & Ap & - \\
\hline 84 & Erigeron annuus (L.) Pers. & Asteraceae & + & + & + & $\mathrm{H}, \mathrm{T}$ & $\mathrm{Kn}$ & - \\
\hline 85 & Erophila verna (L.) Chevall. & Brassicaceae & + & & & $\mathrm{T}$ & Ap & - \\
\hline 86 & Euonymus europaea L. & Celastraceae & + & & & $\mathrm{N}$ & Ap & Rham-Pru \\
\hline 87 & Eupatorium cannabinum $\mathrm{L}$. & Asteraceae & + & + & + & $\mathrm{H}$ & Ap & Artemi \\
\hline 88 & Euphorbia cyparissias L. & Euphorbiaceae & + & + & + & $\mathrm{H}$ & Ap & Fest-Brom \\
\hline 89 & Euphorbia esula L. & Euphorbiaceae & + & & + & $\mathrm{H}$ & Ap & - \\
\hline 90 & Euphorbia platyphyllos L. & Euphorbiaceae & + & & + & $\mathrm{H}$ & Ap & - \\
\hline 91 & Euphrasia rostkoviana Hayne & Scrophulariaceae & + & + & + & $\mathrm{T}$ & Sp & Mol-Arr \\
\hline 92 & Fagus sylvatica $\mathrm{L}$. & Fagaceae & & + & + & M & $\mathrm{Sp}$ & Que-Fag \\
\hline 93 & Fallopia convolvulus (L.) Á. Löve & Polygonaceae & + & & + & $\mathrm{T}$ & Arch & Stel med \\
\hline 94 & Festuca arundinacea Schreb. & Poaceae & & & + & $\mathrm{H}$ & Ap & Mol-Arr \\
\hline 95 & Festuca gigantea (L.) Vill. & Poaceae & & & + & $\mathrm{H}$ & Ap & Que-Fag \\
\hline 96 & Festuca pratensis Huds. & Poaceae & + & + & + & $\mathrm{H}$ & Ap & Mol-Arr \\
\hline 97 & Festuca rubra L.s.s. & Poaceae & + & + & + & $\mathrm{H}$ & Ap & Mol-Arr \\
\hline 98 & Filipendula ulmaria (L.) Maxim. & Rosaceae & & & + & $\mathrm{H}$ & Sp & Mol-Arr \\
\hline 99 & Fragaria vesca L. & Rosaceae & + & + & + & $\mathrm{H}$ & $\mathrm{Sp}$ & Epilob \\
\hline 100 & Fragaria viridis Duchesne & Rosaceae & + & + & + & $\mathrm{H}$ & Sp & Trif-Ger \\
\hline 101 & Fraxinus excelsior $\mathrm{L}$. & Oleaceae & + & + & & M & Ap & Que-Fag \\
\hline 102 & Fumaria officinalis L. & Fumariaceae & & + & & $\mathrm{T}$ & Arch & Stel med \\
\hline 103 & Galeopsis pubescens Besser & Lamiaceae & & & + & $\mathrm{T}$ & Ap & Artemi \\
\hline 104 & Galeopsis tetrahit L. & Lamiaceae & & & + & $\mathrm{T}$ & Ap & Stel med \\
\hline 105 & Galium aparine L. & Rubiaceae & & & + & $\mathrm{T}$ & Ap & Artemi \\
\hline 106 & Galium mollugo L. s. str. & Rubiaceae & + & + & + & $\mathrm{H}$ & Ap & Mol-Arr \\
\hline 107 & Galium verum $\mathrm{L}$. & Rubiaceae & + & + & + & $\mathrm{H}$ & Ap & Trif-Ger \\
\hline 108 & Gentiana cruciata L. [S] & Gentianaceae & + & & & $\mathrm{H}$ & $\mathrm{Sp}$ & Fest-Brom \\
\hline 109 & Geranium dissectum L. & Geraniaceae & & & + & $\mathrm{T}$ & Arch & Stel med \\
\hline
\end{tabular}




\begin{tabular}{|c|c|c|c|c|c|c|c|c|}
\hline \multirow{2}{*}{ No. } & \multirow{2}{*}{ Name of species } & \multirow{2}{*}{ Family } & \multicolumn{3}{|c|}{ Locality } & \multirow{2}{*}{$\mathrm{LF}$} & \multirow{2}{*}{$\mathrm{GHg}$} & \multirow{2}{*}{ PSg } \\
\hline & & & $\mathrm{W}$ & $\mathrm{O}$ & $\mathrm{R}$ & & & \\
\hline 110 & Geranium palustre L. & Geraniaceae & + & & & $\mathrm{H}$ & $\mathrm{Sp}$ & Mol-Arr \\
\hline 111 & Geranium pratense L. & Geraniaceae & + & & & $\mathrm{H}$ & Ap & Mol-Arr \\
\hline 112 & Glechoma hederacea L. & Lamiaceae & & + & & G & Ap & Artemi \\
\hline 113 & Heracleum sphondylium L. s. str. & Apiaceae & + & + & + & $\mathrm{H}$ & Ap & Mol-Arr \\
\hline 114 & Hieracium bauhinii Schult. & Asteraceae & + & + & & $\mathrm{H}$ & Sp & Fest-Brom \\
\hline 115 & Hieracium pilosella L. & Asteraceae & & + & & $\mathrm{H}$ & Ap & Nard-Cal \\
\hline 116 & Holcus lanatus L. & Poaceae & & & + & $\mathrm{H}$ & Ap & Mol-Arr \\
\hline 117 & Holcus mollis L. & Poaceae & & + & + & G, $\mathrm{H}$ & Ap & Que rob \\
\hline 118 & Humulus lupulus L. & Cannabaceae & + & & & $\mathrm{H}$ & Ap & - \\
\hline 119 & Hypericum maculatum Crantz & Hypericaceae & & & + & $\mathrm{H}$ & Sp & Nard-Cal \\
\hline 120 & Hypericum perforatum $\mathrm{L}$. & Hypericaceae & + & + & + & $\mathrm{H}$ & Ap & - \\
\hline 121 & Inula salicina $\mathrm{L}$ & Asteraceae & & + & & $\mathrm{H}$ & Sp & Mol-Arr \\
\hline 122 & Juncus conglomeratus L. & Juncaceae & + & & + & $\mathrm{H}$ & Ap & Nard-Cal \\
\hline 123 & Juncus effusus L. & Juncaceae & + & & & $\mathrm{H}$ & Ap & Mol-Arr \\
\hline 124 & Juncus inflexus $\mathrm{L}$. & Juncaceae & + & & + & $\mathrm{H}$ & Ap & Mol-Arr \\
\hline 125 & Knautia arvensis (L.) J.M. Coult. & Dipsacaceae & + & + & + & $\mathrm{H}$ & Ap & Mol-Arr \\
\hline 126 & Lactuca serriola $\mathrm{L}$. & Asteraceae & + & & & $\mathrm{H}$ & Arch & Stel med \\
\hline 127 & Larix decidua Mill. & Pinaceae & + & & & M & Ap & - \\
\hline 128 & Lathyrus pratensis $\mathrm{L}$. & Fabaceae & + & + & + & $\mathrm{H}$ & Ap & Mol-Arr \\
\hline 129 & Lathyrus tuberosus L. & Fabaceae & + & + & + & $\mathrm{H}$ & Arch & Stel med \\
\hline 130 & Leontodon hispidus L. & Asteraceae & + & + & + & $\mathrm{H}$ & $\mathrm{Sp}$ & Mol-Arr \\
\hline 131 & Lepidium campestre (L.) R. Br. & Brassicaceae & + & + & & $\mathrm{T}$ & Arch & - \\
\hline 132 & Leucanthemum vulgare Lam. s. str. & Asteraceae & + & + & + & $\mathrm{H}$ & Ap & Mol-Arr \\
\hline 133 & Ligustrum vulgare $\mathrm{L}$. & Oleaceae & + & & & $\mathrm{H}$ & Ap & Rham-Pru \\
\hline 134 & Linaria vulgaris Mill. & Scrophulariaceae & + & & + & G & Ap & Artemi \\
\hline 135 & Linum catharticum $\mathrm{L}$. & Linaceae & + & + & + & $\mathrm{T}$ & $\mathrm{Sp}$ & - \\
\hline 136 & Lolium multiflorum Lam. & Poaceae & & & + & $\mathrm{H}, \mathrm{T}$ & $\mathrm{Kn}$ & - \\
\hline 137 & Lolium perenne L. & Poaceae & & + & + & $\mathrm{H}$ & Ap & Mol-Arr \\
\hline 138 & Lotus corniculatus L. & Fabaceae & + & + & + & $\mathrm{H}$ & Ap & Mol-Arr \\
\hline 139 & Luzula campestris (L.) DC. & Juncaceae & & + & & $\mathrm{H}$ & Sp & Nard-Cal \\
\hline 140 & Lychnis flos-cuculi L. & Caryophyllaceae & & & + & $\mathrm{H}$ & Sp & Mol-Arr \\
\hline 141 & Lycopus europaeus L. & Lamiaceae & & & + & $\mathrm{H}$ & $\mathrm{Sp}$ & Aln glu \\
\hline 142 & Lysimachia nummularia L. & Primulaceae & & & + & $\mathrm{C}$ & $\mathrm{Sp}$ & Mol-Arr \\
\hline 143 & Lysimachia vulgaris $\mathrm{L}$. & Primulaceae & & + & + & $\mathrm{H}$ & $\mathrm{Sp}$ & Mol-Arr \\
\hline 144 & Lythrum salicaria L. & Lythraceae & & & + & $\mathrm{H}$ & $\mathrm{Sp}$ & Mol-Arr \\
\hline 145 & Medicago falcata L. & Fabaceae & + & + & + & $\mathrm{H}$ & Ap & Trif-Ger \\
\hline 146 & Medicago lupulina L. & Fabaceae & + & + & & $\mathrm{T}$ & Ap & - \\
\hline 147 & Medicago sativa L. s. str. & Fabaceae & + & & & $\mathrm{H}$ & $\mathrm{Kn}$ & - \\
\hline 148 & Medicago $\times$ varia Martyn & Fabaceae & + & + & & $\mathrm{H}$ & $\mathrm{Kn}$ & - \\
\hline 149 & Melampyrum arvense $\mathrm{L}$. & Scrophulariaceae & + & + & & $\mathrm{T}$ & $\mathrm{Sp}$ & Fest-Brom \\
\hline 150 & Melampyrum nemorosum L. & Scrophulariaceae & + & + & & $\mathrm{T}$ & $\mathrm{Sp}$ & Trif-Ger \\
\hline 151 & Melandrium album (Mill.) Garcke & Caryophyllaceae & + & & & $\mathrm{T}$ & Ap & Artemi \\
\hline 152 & Melilotus alba Medik. & Fabaceae & + & + & & $\mathrm{T}$ & Ap & Artemi \\
\hline 153 & Melilotus officinalis (L.) Pall. & Fabaceae & + & + & + & $\mathrm{T}$ & Ap & Artemi \\
\hline 154 & Mentha arvensis $\mathrm{L}$. & Lamiaceae & + & + & + & G & Ap & - \\
\hline 155 & Mentha longifolia (L.) L. & Lamiaceae & + & + & + & $\mathrm{H}$ & Ap & Mol-Arr \\
\hline 156 & Myosotis palustris (L.) L. emend. Rchb. & Boraginaceae & & & + & $\mathrm{H}$ & Sp & Mol-Arr \\
\hline 157 & Nardus stricta L. & Poaceae & & + & & $\mathrm{H}$ & $\mathrm{Sp}$ & Nard-Cal \\
\hline 158 & Odontites serotina (Lam.) Rchb. s. str. & Scrophulariaceae & + & & + & $\mathrm{T}$ & $S p$ & - \\
\hline 159 & Ononis arvensis L. & Fabaceae & + & + & + & $\mathrm{H}$ & Ap & - \\
\hline 160 & Origanum vulgare $\mathrm{L}$. & Lamiaceae & + & + & + & $\mathrm{C}, \mathrm{H}$ & Sp & Trif-Ger \\
\hline 161 & Padus avium Mill. & Rosaceae & + & & & M & Ap & Que-Fag \\
\hline 162 & Papaver rhoeas $\mathrm{L}$. & Papaveraceae & + & + & & $\mathrm{T}$ & Arch & Stel med \\
\hline 163 & Pastinaca sativa $\mathrm{L}$. & Apiaceae & + & + & & $\mathrm{H}$ & Ap & Artemi \\
\hline 164 & Phalaris arundinacea $\mathrm{L}$. & Poaceae & & & + & $\mathrm{H}$ & Ap & Phragm \\
\hline
\end{tabular}




\begin{tabular}{|c|c|c|c|c|c|c|c|c|}
\hline \multirow{2}{*}{ No. } & \multirow{2}{*}{ Name of species } & \multirow{2}{*}{ Family } & \multicolumn{3}{|c|}{ Locality } & \multirow{2}{*}{ LF } & \multirow{2}{*}{ GHg } & \multirow{2}{*}{ PSg } \\
\hline & & & $\mathrm{W}$ & $\mathrm{O}$ & $\mathrm{R}$ & & & \\
\hline 165 & Phleum pratense $\mathrm{L}$. & Poaceae & & + & + & $\mathrm{H}$ & Ap & Mol-Arr \\
\hline 166 & Picris hieracioides L. & Asteraceae & + & + & + & $\mathrm{H}$ & Ap & Artemi \\
\hline 167 & Pimpinella major (L.) Huds. & Apiaceae & + & + & + & $\mathrm{H}$ & $\mathrm{Sp}$ & Mol-Arr \\
\hline 168 & Pimpinella saxifraga L. s. str. & Apiaceae & + & + & + & $\mathrm{H}$ & Ap & - \\
\hline 169 & Pinus sylvestris $\mathrm{L}$. & Pinaceae & + & + & + & M & Ap & Vac-Pice \\
\hline 170 & Plantago lanceolata $\mathrm{L}$. & Plantaginaceae & + & + & + & $\mathrm{H}$ & Ap & Mol-Arr \\
\hline 171 & Plantago major L. s. str. & Plantaginaceae & & & + & $\mathrm{H}$ & Ap & Mol-Arr \\
\hline 172 & Plantago media L. & Plantaginaceae & + & + & + & $\mathrm{H}$ & Ap & Fest-Brom \\
\hline 173 & Poa compressa $\mathrm{L}$. & Poaceae & + & + & + & $\mathrm{H}$ & Ap & Fest-Brom \\
\hline 174 & Poa nemoralis L. & Poaceae & + & & & $\mathrm{H}$ & Ap & Que-Fag \\
\hline 175 & Poa pratensis $\mathrm{L}$. & Poaceae & + & + & + & $\mathrm{H}$ & Ap & Mol-Arr \\
\hline 176 & Poa trivialis $\mathrm{L}$. & Poaceae & & & + & $\mathrm{H}$ & Ap & Mol-Arr \\
\hline 177 & Polygala comosa Schkuhr & Polygalaceae & + & + & & $\mathrm{H}$ & Sp & - \\
\hline 178 & Polygonum aviculare L. & Polygonaceae & & & + & $\mathrm{T}$ & Ap & Stel med \\
\hline 179 & Polygonum hydropiper L. & Polygonaceae & & & + & $\mathrm{T}$ & Ap & Bident \\
\hline 180 & Populus tremula L. & Salicaceae & + & + & + & M & Ap & Epilob \\
\hline 181 & Potentilla anserina L. & Rosaceae & & & + & $\mathrm{H}$ & Ap & Mol-Arr \\
\hline 182 & Potentilla argentea $\mathrm{L}$. & Rosaceae & + & & & $\mathrm{H}$ & Ap & Koel-Cory \\
\hline 183 & Potentilla erecta (L.) Raeusch. & Rosaceae & + & + & & $\mathrm{H}$ & $\mathrm{Sp}$ & Nard-Cal \\
\hline 184 & Potentilla recta $\mathrm{L}$ & Rosaceae & + & & & $\mathrm{H}$ & Sp & - \\
\hline 185 & Potentilla reptans $\mathrm{L}$. & Rosaceae & + & + & + & $\mathrm{H}$ & Ap & Mol-Arr \\
\hline 186 & Primula veris $\mathrm{L}$. & Primulaceae & & + & & $\mathrm{H}$ & $\mathrm{Sp}$ & Que-Fag \\
\hline 187 & Prunella vulgaris $\mathrm{L}$. & Lamiaceae & & & + & $\mathrm{H}$ & Ap & Mol-Arr \\
\hline 188 & Prunus spinosa $\mathrm{L}$. & Rosaceae & + & + & + & $\mathrm{N}$ & Ap & Rham-Pru \\
\hline 189 & Pyrus pyraster (L.) Burgsd. & Rosaceae & + & + & + & M & Ap & Rham-Pru \\
\hline 190 & Quercus robur $\mathrm{L}$. & Fagaceae & + & + & + & M & $\mathrm{Sp}$ & Que-Fag \\
\hline 191 & Ranunculus acris L. & Ranunculaceae & + & + & + & $\mathrm{H}$ & Ap & Mol-Arr \\
\hline 192 & Ranunculus auricomus L. s. 1. & Ranunculaceae & + & + & & $\mathrm{H}$ & Sp & Que-Fag \\
\hline 193 & Ranunculus polyanthemos L. & Ranunculaceae & + & + & + & $\mathrm{H}$ & Sp & Que-Fag \\
\hline 194 & Ranunculus repens L. & Ranunculaceae & + & + & + & $\mathrm{H}$ & Ap & Mol-Arr \\
\hline 195 & Rhinanthus serotinus (Schönh.) Oborný & Scrophulariaceae & & + & & $\mathrm{T}$ & Arch & Mol-Arr \\
\hline 196 & Rosa canina $\mathrm{L}$ & Rosaceae & + & + & + & $\mathrm{N}$ & Ap & Rham-Pru \\
\hline 197 & Rubus caesius L. & Rosaceae & + & + & + & $\mathrm{N}$ & Ap & - \\
\hline 198 & Rubus hirtus Waldst. et Kit. agg. & Rosaceae & & & + & $\mathrm{N}$ & Ap & Vac-Pice \\
\hline 199 & Rubus idaeus L. & Rosaceae & & & + & $\mathrm{N}$ & Ap & Epilob \\
\hline 200 & Rumex acetosa $\mathrm{L}$. & Polygonaceae & & & + & $\mathrm{H}$ & Ap & Mol-Arr \\
\hline 201 & Rumex obtusifolius L. & Polygonaceae & & + & + & $\mathrm{H}$ & Ap & Artemi \\
\hline 202 & Salix caprea $\mathrm{L}$. & Salicaceae & + & + & + & $\mathrm{N}$ & Ap & Epilob \\
\hline 203 & Salix cinerea $\mathrm{L}$. & Salicaceae & & & + & $\mathrm{N}$ & Ap & Aln glu \\
\hline 204 & Salix purpurea L. & Salicaceae & + & & & $\mathrm{N}$ & Ap & Sal purp \\
\hline 205 & Salvia glutinosa $\mathrm{L}$. & Lamiaceae & & & + & $\mathrm{H}$ & $\mathrm{Sp}$ & Que-Fag \\
\hline 206 & Salvia verticillata $\mathrm{L}$. & Lamiaceae & + & + & + & $\mathrm{H}$ & $\mathrm{Sp}$ & Fest-Brom \\
\hline 207 & Sambucus ebulus L. & Caprifoliaceae & + & + & & $\mathrm{H}$ & Ap & Artemi \\
\hline 208 & Sambucus nigra L. & Caprifoliaceae & + & + & + & $\mathrm{N}$ & Ap & Epilob \\
\hline 209 & Sanguisorba minor Scop. s. str. & Rosaceae & + & + & & $\mathrm{H}$ & Sp & - \\
\hline 210 & Saponaria officinalis L. & Caryophyllaceae & + & & & $\mathrm{H}$ & Ap & Artemi \\
\hline 211 & Scirpus sylvaticus L. & Cyperaceae & & & + & G & Sp & Mol-Arr \\
\hline 212 & Sedum acre $\mathrm{L}$. & Crassulaceae & + & + & & $\mathrm{C}$ & Ap & Koel-Cory \\
\hline 213 & Sedum maximum (L.) Hoffm. & Crassulaceae & & + & & $\mathrm{G}, \mathrm{H}$ & Ap & - \\
\hline 214 & Sedum sexangulare $\mathrm{L}$. & Crassulaceae & & + & & $\mathrm{C}$ & Sp & Koel-Cory \\
\hline 215 & Senecio jacobaea $\mathrm{L}$. & Asteraceae & + & + & + & $\mathrm{H}$ & Ap & - \\
\hline 216 & $\begin{array}{l}\text { Senecio ovatus (P. Gaertn., B. Mey. } \\
\text { et Scherb.) Willd. }\end{array}$ & Asteraceae & & & + & $\mathrm{H}$ & Ap & Epilob \\
\hline 217 & Setaria pumila (Poir.) Roem. et Schult. & Poaceae & & + & & $\mathrm{T}$ & Arch & Stel med \\
\hline 218 & Silene vulgaris (Moench) Garcke & Caryophyllaceae & & + & & $\mathrm{H}$ & Ap & Stel med \\
\hline 219 & Sinapis arvensis $\mathrm{L}$ & Brassicaceae & + & & & $\mathrm{T}$ & Arch & Stel med \\
\hline
\end{tabular}




\begin{tabular}{|c|c|c|c|c|c|c|c|c|}
\hline \multirow{2}{*}{ No. } & \multirow{2}{*}{ Name of species } & \multirow{2}{*}{ Family } & \multicolumn{3}{|c|}{ Locality } & \multirow{2}{*}{$\mathrm{LF}$} & \multirow{2}{*}{$\mathrm{GHg}$} & \multirow{2}{*}{ PSg } \\
\hline & & & $\mathrm{W}$ & $\mathrm{O}$ & $\mathrm{R}$ & & & \\
\hline 220 & Solidago gigantea Aiton & Asteraceae & + & + & + & $\mathrm{G}, \mathrm{H}$ & $\mathrm{Kn}$ & Artemi \\
\hline 221 & Solidago virgaurea L. s. str. & Asteraceae & + & + & + & $\mathrm{H}$ & $\mathrm{Sp}$ & Que rob \\
\hline 222 & Stachys palustris L. & Lamiaceae & + & & + & G & Ap & Mol-Arr \\
\hline 223 & Stellaria graminea L. & Caryophyllaceae & & & + & $\mathrm{H}$ & Ap & - \\
\hline 224 & Symphytum officinale L. & Boraginaceae & + & + & + & $\mathrm{H}$ & Ap & - \\
\hline 225 & Tanacetum vulgare $\mathrm{L}$. & Asteraceae & + & + & + & $\mathrm{H}$ & Ap & Artemi \\
\hline 226 & Thlaspi arvense L. & Brassicaceae & & + & & $\mathrm{T}, \mathrm{H}$ & Arch & Stel med \\
\hline 227 & Thymus pulegioides L. & Lamiaceae & + & + & + & $\mathrm{C}$ & $\mathrm{Sp}$ & - \\
\hline 228 & Torilis japonica (Houtt.) DC. & Apiaceae & + & & + & $\mathrm{H}, \mathrm{T}$ & Ap & Artemi \\
\hline 229 & Tragopogon pratensis L. & Asteraceae & & + & + & $\mathrm{H}$ & Ap & Mol-Arr \\
\hline 230 & Trifolium arvense L. & Fabaceae & & & + & $\mathrm{T}$ & Ap & Koel-Cory \\
\hline 231 & Trifolium campestre Schreb. & Fabaceae & + & & + & $\mathrm{T}$ & Ap & Koel-Cory \\
\hline 232 & Trifolium dubium Sibth. & Fabaceae & & + & & $\mathrm{T}$ & Ap & Mol-Arr \\
\hline 233 & Trifolium hybridum L. & Fabaceae & + & & + & $\mathrm{H}$ & Ap & Mol-Arr \\
\hline 234 & Trifolium medium L. & Fabaceae & + & + & + & $\mathrm{H}$ & Ap & Trif-Ger \\
\hline 235 & Trifolium montanum L. & Fabaceae & & + & & $\mathrm{H}$ & $\mathrm{Sp}$ & Mol-Arr \\
\hline 236 & Trifolium pratense $\mathrm{L}$. & Fabaceae & + & & + & $\mathrm{H}$ & Ap & Mol-Arr \\
\hline 237 & Trifolium repens $\mathrm{L}$. & Fabaceae & & + & + & $\mathrm{H}$ & Ap & Mol-Arr \\
\hline 238 & Trisetum flavescens (L.) P. Beauv. & Poaceae & + & & & $\mathrm{H}$ & $\mathrm{Sp}$ & Mol-Arr \\
\hline 239 & Tussilago farfara L. & Asteraceae & + & & + & G & Ap & Artemi \\
\hline 240 & Urtica dioica $\mathrm{L}$. & Urticaceae & + & + & + & $\mathrm{H}$ & Ap & Artemi \\
\hline 241 & Valeriana officinalis L. & Valerianaceae & + & & & $\mathrm{H}$ & $\mathrm{Sp}$ & Mol-Arr \\
\hline 242 & Verbascum nigrum L. & Scrophulariaceae & + & + & & $\mathrm{H}$ & $\mathrm{Sp}$ & Epilob \\
\hline 243 & Veronica chamaedrys L. & Scrophulariaceae & + & + & + & $\mathrm{C}$ & Ap & - \\
\hline 244 & Veronica serpyllifolia L. & Scrophulariaceae & + & & & $\mathrm{H}$ & Ap & Mol-Arr \\
\hline 245 & Viburnum opulus L. & Caprifoliaceae & & + & + & $\mathrm{N}$ & $\mathrm{Sp}$ & Rham-Pru \\
\hline 246 & Vicia cracca L. & Fabaceae & + & + & + & $\mathrm{H}$ & Ap & Mol-Arr \\
\hline 247 & Vicia grandiflora Scop. & Fabaceae & & + & & $\mathrm{T}$ & $\mathrm{Kn}$ & - \\
\hline 248 & Vicia hirsuta (L.) Gray & Fabaceae & & + & & $\mathrm{T}$ & Arch & Stel med \\
\hline 249 & Vicia sepium L. & Fabaceae & + & + & & $\mathrm{H}$ & $\mathrm{Sp}$ & Trif-Ger \\
\hline 250 & Viola canina $\mathrm{L}$. & Violaceae & & + & & $\mathrm{H}$ & $\mathrm{Sp}$ & Nard-Cal \\
\hline 251 & Viola odorata L. & Violaceae & + & & & $\mathrm{H}$ & Ap & Artemi \\
\hline 252 & Viola riviniana $\mathrm{Rchb}$. & Violaceae & & + & & $\mathrm{H}$ & $\mathrm{Sp}$ & Que-Fag \\
\hline
\end{tabular}

Explanations

Locality: W - Wojkówka, O - Odrzykoń, R - Rzepnik.

Protection of species: [S] - strictly protected species, [P] - partially protected species.

PSg (phytosociological group): Agr-rep - Agropyretea intermedio-repentis, Aln glu - Alnetea glutinosae, Artemi - Artemisietea, Bident - Bidentetea tripartiti, Epilob - Epilobietea angustifolii, Fest-Brom - Festuco-Brometea, Koel-Cory - Koelerio glaucae-Corynephoretea canescentis, Mol-Arr - Molinio-Arrhenatheretea, Nard-Cal - Nardo-Callunetea, Phragm - Phragmitetea, Que-Fag - Querco-Fagetea, Que rob - Quercetea robori-petraeae, Rham-Pru - Rhamno-Prunetea, Sal purp - Salicetea purpureae, Stel med - Stellarietea mediae, Trif-Ger - Trifolio-Geranietea sanguinei, Vac-Pice - Vaccinio-Piceetea.

LF (life forms): M - megaphanerophyte, $\mathrm{N}$ - nanophanerophyte, $\mathrm{C}$ - herbaceous chamaephyte, $\mathrm{H}$ - hemicryptophyte, G - geophyte, T therophyte.

GHg (geographic-historical groups): Ap - apophytes (spontaneophytes synanthropic), Arch - archaeophytes, Kn - kenophytes, Sp spontaneophytes nonsynanthrophic.

phytes $(58.7 \%$ of the total flora) and 80 spontaneophytes $(31.8 \%)$. Anthropophytes were dominated by archeophytes (17 species $-6.7 \%$ of the flora) represented by common weeds: Consolida regalis, Lathyrus tuberosus, Papaver rhoeas, Vicia hirsuta, and Setaria pumila. The other anthropophytes were represented by seven kenophytes $(2.8 \%)$ (Table 3 ).

The analysis of the phytosociological groups indicates that the investigated area is represented by species characteristic for 17 classes (Table 4). The primary aim of the conservation of the area is sustenance of the thermophilic meadow complexes (Mo-
linio-Arrhenatheretea) with fragments of xerothermic grasslands (Festuco-Brometea) and margin communities (Trifolio-Geranietea). Species typical of xerothermic grasslands (Festuco-Brometea) accounted for $5.6 \%$ (14 species). The most frequent taxa included Centaurea scabiosa, Allium oleraceum, Carlina vulgaris, Euphorbia cyparissias, Salvia verticillata, Plantago media, and Melampyrum arvense. A slightly lower proportion was reported for species of thermophilic margin communities (Trifolio-Geranietea) 4.7\% (12 species) such as Medicago falcata, Agrimonia eupatoria, Clinopodium vulgare, Coronilla varia, Origanum vulgare, and Galium 
Table 2. Share of life forms in the flora of Natura 2000 "Łąki nad Wojkówką"

\begin{tabular}{lcc}
\hline \multicolumn{1}{c}{ Life form } & $\begin{array}{c}\text { Number of } \\
\text { species }\end{array}$ & $\%$ \\
\hline Megaphanerophyte & 14 & 5.6 \\
Nanophanerophyte & 13 & 5.1 \\
Chamaephyte & 6 & 2.4 \\
Hemicryptophyte & 160 & 63.5 \\
Geophyte & 22 & 8.7 \\
Therophyte & 37 & 14.7 \\
\hline Total & 252 & 100 \\
\hline
\end{tabular}

Table 3. Geographic-historical status groups in the flora of Natura 2000 "Łąki nad Wojkówką"

\begin{tabular}{lcr}
\hline \multicolumn{1}{c}{$\begin{array}{c}\text { Geographic-historical } \\
\text { status }\end{array}$} & $\begin{array}{c}\text { Number of } \\
\text { species }\end{array}$ & $\%$ \\
\hline Apophytes & 148 & 58.7 \\
Spontaneophytes & 80 & 31.8 \\
Archaeophytes & 17 & 6.7 \\
Kenophytes & 7 & 2.8 \\
\hline Total & 252 & 100 \\
\hline
\end{tabular}

verum. Xerothermic grassland communities together with thermophilic scrubs and shrub communities formed a dynamic mosaic, which is an ecologically indivisible unit. The highest share in the analysed flora was exhibited by fresh meadow species from the class Molinio-Arrhenatheretea (66 species - 26.2\%). The meadow structure was dominated by high grasses with the predominant Arrhenatherum elatius species. Species of the order Arrhenetheretalia elatioris, such as Galium mollugo, Knautia arvensis, Lotus corniculatus, Pimpinella major, Plantago lanceolata, Achillea millefolium, Daucus carota, and Leucanthemum vulgare, were particularly abundant; their presence is an indication of the thermophilic nature of these meadows.

Notably, other communities were represented by species from the class Rhamno-Prunetea accompany-

Table 4. Share of phytosociological groups in the flora of Natura 2000 "Łąki nad Wojkówką"

\begin{tabular}{lcr}
\hline \multicolumn{1}{c}{ Phytosociological units } & $\begin{array}{c}\text { Number of } \\
\text { species }\end{array}$ & $\%$ \\
\hline Bidentetea tripartiti & 2 & 0.8 \\
Stellarietea mediae & 15 & 6 \\
Epilobietea angustifolii & 10 & 4 \\
Artemisietea vulgaris & 30 & 12 \\
Agropyretea intermedio-repentis & 3 & 1.1 \\
Phragmitetea & 1 & 0.4 \\
Koelerio glauce-Corynephoretea canescentis & 6 & 2.4 \\
Molinio-Arrhenatheretea & 66 & 26.2 \\
Festuco-Brometea & 14 & 5.6 \\
Nardo-Callunetea & 11 & 4.3 \\
Trifolio-Geranietea sanguinei & 12 & 4.7 \\
Rhamno-Prunetea & 9 & 3.6 \\
Salicetea purpureae & 1 & 0.4 \\
Alnetea glutinosae & 2 & 0.8 \\
Vaccinio-Piceetea & 3 & 1.2 \\
Quercetea robori-petraeae & 2 & 0.8 \\
Querco-Fagetea & 23 & 9.1 \\
Inne & 42 & 16.6 \\
\hline Total & 252 & 100 \\
\hline
\end{tabular}

ing xerothermic grasslands and accounting for 3.6\% (9 species). The most frequent of these were Prunus spinosa, Crataegus monogyna, Cornus sanguinea, and Rosa canina. Ruderal species from the class Artemisietea vulgaris, which were primarily noted at the border of the area near roads, paths, fallows, and disturbed sites, had a significant share (30 species $-12 \%$ ) in the flora. Such species as Artemisia vulgaris, Chaerophyllum aromaticum, Cirsium arvense, Eupatorium cannabinum, Melilotus officinalis, Picris hieracioides, Tanacetum vulgare, and Urtica dioica were found in all the three localities. Forest species from the class Querco-Fagetea (23 species $-9.1 \%$ ) associated with the forest ecosystems adjacent to the study area were also reported. Plant species with undefined phytosociological affiliation accounted for $16.6 \%$ of the analysed flora.

The floristic inventory of the "Łąki nad Wojkówką" revealed occurrence of only three legally protected species, i.e. Dianthus armeria and Gentiana cruciata, which are under strict protection, and the partially protected Centaurium erythraea (RozPORZĄDZENIE... 2014). However, the Gentianella ciliata species reported by Mróz \& Rogata (2011) was not found.

\section{DISCUSSION}

The great richness of plant species in the analysed area is associated with the diversity of habitats in terms of wetness, terrain features, and the intensity and type of anthropogenic factors. The geological structure also exerts a significant effect on the occurrence of thermophilic vegetation. The geological formations of the analysed area are rich in calcium carbonate, which contributes to development of habitats of xerothermic plants (Wójcik et al. 2014). The area is overgrown by grassland, meadow, forest, scrub, and wet meadow species as well as species typical of segetal and synanthropic habitats. The degree of isolation of the individual study objects, which are located at a distance of a few to several dozen kilometres, is also important. They constitute small enclaves of xerothermic plants surrounded by forests, scrubs, and arable fields.

The study area exhibits a high proportion of species from the class Molinio-Arrhenatheretea, while the share of species characteristic for the classes Festuco-Brometea and Trifolio-Geranietea is substantially lower. The results of the phytosociological analyses confirm the occurrence of transitional phytocoenoses, which resemble thermophilic meadows, xerothermic grasslands, and margin communities (Ziaja \& Wójcik 2014). Similar communities were distinguished by Kucharzyk (2010) and Barabasz-Krasny (2011) from the Przemyskie Foothills and by Wójcik \& Piątek (2015) from the Strzyżowskie Foothills.

In the Carpathian Foothills, thermophilic grasslands are rare communities occurring primarily on 
slopes with southern exposure in the valleys of large rivers. They occupy small areas with unspecified syntaxonomic affiliation and with a large share of species from the classes Trifolio-Geranietea and Molinio-Arrhenatheretea (TowPASz 1990, OKLejeWicz 1996, TrĄBA et al. 2006).

The floristic values of the analysed area are evidenced by the natural character of the flora, which confirms the high share of native species $(90.5 \%)$. The proportion of alien species, which accounted for $9.5 \%$ of the analysed flora, is negligible. The management of the area, in particular of the quarries, promoted encroachment of alien elements in our flora. Kenophytes can mainly be found in disturbed sites, e.g. at roadsides or quarries; Solidago gigantea and Erigeron annuus were the only species growing in all the three localities and they were common in the study area. The presence of the agrocoenoses in the area neighbourhood promoted occurrence of archeophytes.

A major threat to xerothermic grasslands is the abandonment of traditional management, which leads to progression of secondary succession, changes in the community structure, increased wetness, and transformation of grasslands into meadow and scrub communities (Bąba 2004, Perzanowska \& KuJaWA-PaWlaczyK 2004, BarańsKa \& JermaczeK 2009). This process results in a decline in the number and, in some sites, total disappearance of xerothermic species. This may have been the cause of the disappearance of Gentianella ciliata (partially protected species). Abandonment of grassland management leads to initial transformation into thermophilic margin communities from the class Trifolio-Geranietea, and next into thermophilic scrubs (BARAŃSKA \& JERMACZEK 2009).

Similar processes are observed in meadows that transform towards scrub communities after abandonment of management. A rapid decrease in the species richness accompanied by development of expansive grass and dicot species is then observed (KRYSZAK 2004, KrYSZAK \& KrYsZAK 2007, WolaŃSKI \& Rogut 2012). In the analysed area, such processes are especially intensive in Rzepnik, where due to abandonment of management the meadows have been colonised by nitrophilous and expansive species of high perennials (Urtica dioica, Cirsium arvense, Solidago gigantea).

The meadow and xerothermic vegetation in Wojkówka, Odrzykoń, and Rzepnik fully deserves to be protected. Active protection measures should be implemented in order to conserve these thermophilic vegetation stands, which are unique in the Western Carpathians. Mowing and hay removal, as well as grazing practices, will impede the development of expansive perennials and woody vegetation. Therefore, the degree of identification and conservation of xerothermic grassland species is important for effective protection.

\section{CONCLUSIONS}

The Natura 2000 area "Łąki nad Wojkówką" aims at conservation of the communities of xerothermic grasslands and thermophilic meadows, which are rare in the region of the Carpathian Foothills.

The vascular flora comprises 252 species from 49 families and 166 genera. Asteraceae, Poaceae, Fabaceae, Rosaceae, Lamiaceae, and Apiaceae are the richest families.

Native species, i.e. spontaneophytes and apophytes, dominate, which indicates a natural character of the area.

Protected species Dianthus armeria, Gentiana cruciata, Centaurium erythraea were noted; however, Gentianella ciliata was not found during the investigations.

Conservation of the study area necessitates systematic treatments such as mowing, grazing, and felling trees and shrubs that disturb the structure of the ecosystem and contribute to retreat of thermophilic species.

\section{ACKNOWLEDGMENTS}

The authors thank Wacław Bartoszek PhD for identification of Alchemilla monticola and species from the genus Carex.

\section{REFERENCES}

BarabasZ-Krasny B. (2011): Zróżnicowanie roślinności i sukcesja wtórna na odłogach wielkopowierzchniowych Pogórza Przemyskiego. Instytut Botaniki im. W. Szafera, PAN, Kraków.

Barańska K., Jermaczek A. (2009): Poradnik utrzymania i ochrony siedlisk przyrodniczych 6210 - murawy kserotermiczne. Wyd. Klubu Przyrodników, Świebodzin.

BĄBA W. (2004): The species composition and dynamics in well-preserved and restored calcareous xerothermic grasslands (South Poland). Biologia, Bratislava 59(4): 447-456.

Council Directive 92/43/EEC of 21 May 1992 on the conservation of natural habitats and of wild fauna and flora.

KAŹMIERCZAKOWA R. (2004): Kserotermiczne murawy i zarośla Pienińskiego Parku Narodowego. Studia Naturae 49: 277-296.

KondRACKI J. (2011): Geografia regionalna Polski. Wyd. Nauk. PWN, Warszawa.

KornAś J. (1968): Geograficzno-historyczna klasyfikacja roślin synantropijnych. Materiały Zakładu Fitosocjologii Stosowanej UW 25: 33-41.

KRYSZAK A. (2004): Synantropizacja wybranych zbiorowisk łąkowych. Woda-Środowisko-Obszary Wiejskie 4, 1(10): 201-208. 
KRYSZAK J., KRYSZAK A. (2007): Użytkowanie a walory przyrodnicze zbiorowisk łąkowych. Fragmenta Agronomica 24(3): 258-267.

KuchARZYK S. (2010): Murawa kserotermiczna z zawilcem wielkokwiatowym Anemone sylvestris L. na Pogórzu Przemyskim. Chrońmy Przyrodę Ojczystą 66(3): 190-200.

Matuszkiewicz W. (2005): Przewodnik do oznaczania zbiorowisk roślinnych Polski. Wyd. Nauk. PWN, Warszawa.

Mirek Z., Piękoś-Mirkowa H., Zając A., Zając M. (2002): Flowering plants and pteridophytes of Poland. A checklist. Vol. 1. Biodiversity of Poland. Krytyczna lista roślin naczyniowych Polski. T. 1. Różnorodność biologiczna Polski. W. Szafer Institute of Botany, Polish Academy of Science, Kraków.

Mróz K., Rogaza D. (2011): Łąki nad Wojkówką. In: D. Rogała, A. Marcela (eds). Obszary Natura 2000 na Podkarpaciu. Regionalna Dyrekcja Ochrony Środowiska, Rzeszów: 205-207.

ОкLеJEWICZ K. (1996): Charakterystyka geobotaniczna Dołów Jasielsko-Sanockich. Zeszyty Naukowe Uniwersytetu Jagiellońskiego, Prace Botaniczne 27: 1-93.

PAWŁowski B. (1977): Szata roślinna gór polskich. In: W. Szafer, K. Zarzycki (eds). Szata roślinna Polski. T. 2. PWN, Warszawa: 189-252.

Perzanowska J., Kujawa-PawlaczyK J. (2004): Murawy kserotermiczne Festruco-Brometea. In: J. Herbich (ed.). Poradnik ochrony siedlisk i gatunków Natura 2000 - podręcznik metodyczny. T. 3. Ministerstwo Środowiska, Warszawa.

RozPORZĄDZENIE Ministra Środowiska z dnia 9 października 2014 r. w sprawie ochrony gatunkowej roślin. Dz.U. 2014, poz. 1409.

RutKowski L. (2004): Klucz do oznaczania roślin naczyniowych Polski niżowej. Wyd. Nauk. PWN, Warszawa.

TOKARSKA-GuZIK B. (2005): The establishment and spreading of alien plant species (kenophytes) in the Flora of Poland. Wyd. Uniwersytetu Śląskiego, Katowice.

TowPAsz K. (1990): Charakterystyka geobotaniczna Pogórza Strzyżowskiego. Zeszyty Naukowe Uniwersytetu Jagiellońskiego, Rozprawy Habilitacyjne 178 .
Trąba C., Wolański P., OKLejewicz K. (2006): Różnorodność florystyczna wybranych zbiorowisk nieleśnych doliny Sanu. Annales Universitatis Mariae Curie-Skłodowska, Sectio E, 61: 267-275.

Trąba C., Wolański P., Oklejewicz K. (2012): Communities with Brachypodium pinnatum and Bromus erectus in the Wiar and San Valley. Annales Universitatis Mariae Curie-Skłodowska, Sectio C, 67(1): 70-92.

WolAŃSKi P., Rogut K. (2012): Zróżnicowanie florystyczne zbiorowisk nieużytkowanych łąk z rzędu Arrhenatheretalia na Pogórzu Przemyskim. Ekologia i Technika 20(5): 294-305.

Wójcik T., Piątek K. (2015): New locality of Gentiana cruciata L. in the Strzyżowskie Foothills (Western Carpathians). Steciana 19(2): 67-73.

Wóscik T., Ziaja M., Ćwik A. (2014): Potencjał geoturystyczny nieczynnych kamieniołomów Czarnorzecko-Strzyżowskiego Parku Krajobrazowego. Prace Komisji Krajobrazu Kulturowego 26: 155-173.

Zając A. (1979): Pochodzenie archeofitów występujących w Polsce. Zeszyty Naukowe Uniwersytetu Jagiellońskiego, Rozprawy Habilitacyjne 29.

ZAJA̧C M., ZAJĄC A. (1992): A tentative list of segetal and ruderal apophytes in Poland. Zeszyty Naukowe Uniwersytetu Jagiellońskiego, Prace Botaniczne 24: 11-23.

ZająC M., ZajĄC A. (2011): Methodical problems in distinguishing the group of archaeophytes. Synantropizacja $\mathrm{w}$ dobie zmian różnorodności biologicznej. Acta Botanica Silesiaca 6: 55-62.

ZaRZYCKI K., TRZCIŃSKA-TACIK H., RóŻAŃSKI W., SZELĄG Z., Wotek J., Korzeniak U. (2002): Ecological indicator values of vascular plants of Poland. In: $Z$. Mirek (ed.). Biodiversity of Poland. W. Szafer Institute of Botany, Polish Academy of Sciences, Kraków.

ZiAjA M., Wójcik T. (2014): Termophilic plant communieties in Natura 2000 site "Łąki nad Wojkówka” PLH 180051 - Podkarpacie Province. Annales Universitatis Mariae Curie-Skłodowska, Sectio C, 69(1): 59-78.

For citation: ZiajA M., WójciK T. (2015): Vascular flora of the Natura 2000 area "Łąki nad Wojkówką" PLH 180051 (Dynowskie Foothills). Steciana 19(2): 105-114. DOI 10.12657/steciana.019.012 